\title{
Soluble plasma proteins ST2 and CD163 as early biomarkers of nephropathy in Swedish patients with diabetes, 15-34 years of age: a prospective cohort study
}

\author{
My Samuelsson ${ }^{1}$, Jonatan Dereke ${ }^{1}$, Maria K. Svensson ${ }^{2}$, Mona Landin-Olsson ${ }^{1,3}$, Magnus Hillman ${ }^{1^{*}}$ (]) \\ and on the behalf of the DISS Study group
}

\begin{abstract}
Background: The aim of this study was to investigate plasma levels of sST2 and sCD163 to determine whether they at an early stage could predict development of diabetic nephropathy and/or diabetic retinopathy in patients at clinical onset.

Methods: Patients diagnosed with diabetes mellitus at age 15-34 years between 1987 and $1988(n=220)$ were included. Data such as BMI, smoking, HbA1c and islet cell antibodies were collected at time of diagnosis. Within the 10 year follow-up period, 112 patients (51\%) developed following diabetes related complications; retinopathy $(n=91)$, nephropathy $(n=12)$ or both $(n=9)$. Plasma concentrations of sST2 and SCD163 were measured at time of diagnosis and levels compared between different complication groups.

Results: Plasma levels of sST2 were significantly higher in patients who later developed nephropathy $(n=21 ; 1012$ [773-1493] pg/ml) compared to those who did not $(n=199 ; 723$ [449-1084] pg/ml; $p=0.006)$. A tendency for higher plasma levels of SCD163 was observed but not statistically significant $(p=0.058)$.

Conclusions: SST2 and SCD163 show promise as potential biomarkers for the development of nephropathy already at clinical onset. sST2 and/or sCD163 could possibly be part of a biomarker panel aimed to find patients at high risk of developing nephropathy. Both markers need to be investigated in a larger prospective study.
\end{abstract}

Keywords: Diabetes, Nephropathy, Biomarkers, Complications

\section{Background}

Diabetes related long-term complications cause high economical and personal costs and impact quality of life. In many cases these long-term complications can be prevented or delayed if diagnosed and treated in a timely manner [1]. It is clear that in order to administer proper treatment early on, more effective predictive markers of long-term complications are needed. One complication of particular importance is diabetic nephropathy (DN),

\footnotetext{
*Correspondence: Magnus.Hillman@med.lu.se

${ }^{1}$ Department of Clinical Sciences, Diabetes Research Laboratory, Lund University, BMC, B11, 22184 Lund, Sweden

Full list of author information is available at the end of the article
}

a severe microvascular disease, and one of the primary causes of morbidity and mortality amongst patients with diabetes mellitus [2]. Several factors related to DN development have been identified, such as hyperglycemia, hypertension, genetic predisposition and epigenetics, however a simple and effective predictive marker for $\mathrm{DN}$ is yet to be found $[1,3]$. DN is currently treated with lifestyle interventions, intensive blood pressure- and glycemic control, and treatment of dyslipidemia. Despite available therapies there is a progression of the disease $[4,5]$. In addition, some patients develop DN rapidly even with modest hyperglycemia while others never develop $\mathrm{DN}$, in spite of poor glycemic control. This suggests 
genetic traits and molecular mechanisms of the disease that are not yet fully understood.

Diabetic nephropathy can be classified after level of severity; incipient nephropathy (microalbuminuria) and overt nephropathy (macroalbuminuria), the latter being more severe and strongly related to development of renal impairment and end-stage renal disease (ESRD) [6]. Increased urinary excretion of albuminuria is an early clinical manifestation of renal damage or dysfunction in diabetes and is also an important risk factor for progression of renal impairment and development of ESRD [7] as well as an established risk factor for cardiovascular disease (CVD) and all-cause mortality [8]. Microalbuminuria is defined as a urine albumin excretion rate (UAER) of $20-200 \mu \mathrm{g} / \mathrm{min}$ and macroalbuminuria as an UAER of $>200 \mu \mathrm{g} / \mathrm{min}$. Microalbuminuria is generally a sign of glomerular damage and macroalbuminuria is usually accompanied by clinical manifestations of renal impairment such as reduced glomerular filtration rate (GFR) [9] as well as more prominent structural histological lesions in the kidney [10].

The most commonly used biomarkers for identifying renal disease progression today are serum creatinine and estimated GFR (eGFR). The precision is however low and there is need for more sensitive markers for early prediction of nephropathy. Several novel tubular and inflammatory biomarkers have been evaluated as potential predictors for nephropathy, most of which have been investigated in early kidney damage [11]. A couple of urinary tubular biomarkers has been reported to be increased in patients with type 1 diabetes several years before development of DN. Further studies in both urine and blood are required to confirm these findings and to study if the results persist in patients with type 2 diabetes $[12,13]$.

Diabetic retinopathy (DR) is another diabetes related long-term microvascular complication. The retinal vasculature is sensitive to hyperglycemia which induces oxidative stress, an increase in advanced glycation end products (AGEs) and activation of protein kinase $\mathrm{C}$ (PKC) amongst other destructive processes [14]. These underlying changes cause microaneurysms, macular edema and neovascularization impairing vision [15].

Due to the complexity of complication development it is probable that a future biomarker panel consisting of several different proteins and miRNAs could yield better precision than the use of single biomarkers. Thus there is a need to identify potential candidates.

Soluble ST2 (sST2) is a free form of the membrane bound ST2, found on the surface of Th2-cells, mast cells and basophil granulocytes. ST2 is an interleukin-33 (IL33) receptor which upon binding induces Th2 activity and increases Th2 inflammatory responses by inducing chemotaxis in Th2-cells as well as the release of Th2associated cytokines [16]. Presumably sST2 acts as a decoy receptor for IL-33, preventing it from binding to membrane bound ST2 and thereby reducing the biological effect of IL-33 [17]. Plasma levels of sST2 reportedly correlate with diabetic markers such as triglycerides, hepatic function and plasma glucose as well as incidence of diabetes mellitus $[18,19]$. Thus, SST2 may play part in the inflammatory process of diabetes mellitus, although the mechanisms behind this are not yet fully understood. Levels of sST2 have been associated with cardiovascular complications and mortality $[20,21]$ and suggested to have a prognostic value as risk predictor in patients with ambulatory chronic heart failure [22].

Soluble CD163 (sCD163) is the shedded ectodomain of membrane bound CD163, an endocytic receptor found on some monocyte and macrophage populations [23]. The biological function of sCD163 is not entirely understood, although levels of sCD163 rise markedly during inflammation and insulin resistance [24] making it a promising biomarker for macrophage involvement in prediabetes [25]. Furthermore, sCD163 interacts with a ligand known as Tumor Necrosis Factor Weak Inducer of Apoptosis (TWEAK) which have been associated to obesity and type 2 diabetes [26], but also to tissue regeneration after ischemic injury [27]. Increased expression of TWEAK in vitreous samples have been reported in patients with DR [28], however the possible interaction with sCD163 was not investigated. High levels of sCD163 results in an increased risk of type 2 diabetes development [29], maybe as a result of macrophage infiltration to adipose tissue and liver.

The aim of this study was to assess plasma levels of sST2 and sCD163 to determine whether they are associated with development of diabetes related long-term complications (DN and DR) in patients with onset of diabetes as young adults. If so, sST2 and/or sCD163 might serve as early biomarkers or to be included in a panel of biomarkers identifying patients at high risk for development of diabetes related complications and enable a closer monitoring of high risk patients as well as facilitate development of better treatment options to delay or prevent onset of complications.

\section{Methods \\ Study population}

Subjects included in the study were diagnosed with type $1(n=131)$ or type 2 diabetes $(n=89)$ mellitus by their reporting physician according to World Health Organization criteria [30]. All subjects were part of the Diabetes Incidence Study in Sweden (DISS) [31]). A total of 220 participants were included, all diagnosed with diabetes mellitus in 1987-88 at an age of 15-34 years. Out of 
these patients, 112 (51\%) developed the following diabetes related complications within the 8 year follow up period; DR ( $\mathrm{n}=91)$, DN $(\mathrm{n}=12)$ or concomitant DR and $\mathrm{DN}(\mathrm{n}=9)$. The remaining 108 developed neither of the complications and were used as a control group. In this study, the term DN refers to persistent micro- or macroalbuminuria, thus incipient or manifest DN. Persistent microalbuminuria (incipient nephropathy) and macroalbuminuria (overt nephropathy) are both referred to as DN [32]. Identification of patients with DN was performed in two steps as previously reported and patients were not categorized as having DN if other causes for albuminuria were found. The selection and classification of the study population has been described in greater detail elsewhere [31].

\section{Sample handling}

Blood samples were collected in tubes containing EDTA at time of clinical diagnosis of diabetes mellitus. Samples were sent to the laboratory from health care centers and hospitals in Sweden by ordinary mail. Plasma was separated by centrifugation at $2000 \times g$ and stored in $-20{ }^{\circ} \mathrm{C}$ until use. HbA1c levels were measured at local hospitals by ion chromatography and presented in NGSP \% followed by International Federation of Clinical Chemistry (IFCC) units (mmol/mol). Both sCD163 and sST2s are verified as stable in frozen plasma samples and reportedly robust to thawing $[29,33]$.

\section{Laboratory analyses}

Plasma concentrations of sST2 and sCD163 respectively were measured in duplicates by the use of sandwich enzyme-linked immunosorbent assay (ELISA) (R\&D Systems, Minneapolis, Minnesota, USA) DuoSet kit and supplemental DuoSet Ancillary Reagent Kit 2 (R\&D Systems, Minneapolis, Minnesota, USA) according to manufactures instructions. Two in-house plasma controls were added to each run. The sST2 kit used has a reported lower detection limit of $32 \mathrm{pg} / \mathrm{ml}$ [33]. The inter assay variability was calculated to $11.6 \%$ for sST2 and $9.7 \%$ for sCD163. Samples were diluted, sST2 1:3 and sCD163 1:200 respectively. All samples were added in duplicates, subjects and controls alternated on each plate to reduce inter-assay variability. Optical density (OD) was measured at 450 and $580 \mathrm{~nm}$ with a FLUOstar OPTIMA microplate reader (BMG LABTECH, Ortenberg, Germany). A four-parametric logistic regression standard curve was used to read the concentrations.

\section{Statistical analyses}

D'Agostino-Pearson test was used to test for normal distribution of data. Results are presented as mean $\pm \mathrm{SD}$ when normality was accepted and median followed by interquartile range (Q1-Q3) when normality was rejected. Differences between groups were tested with Student's $t$ test for normally distributed data otherwise Mann-Whitney U-test or Kruskal-Wallis H-test were used. Differences in observed and expected frequencies between groups were calculated using $x^{2}$-test. Correlations between variables were determined using Spearman's rank correlation test. $\mathrm{p}$ values below 0.05 were considered as statistically significant. All statistical analyses were performed using $\mathrm{MedCalc}^{\circledR}{ }^{\circledR}$ version 14.12.0 for Windows (MedCalc Software, Ostend, Belgium).

\section{Results}

Clinical and laboratory data in study participants are summarized in Table 1.

Patients who developed DN within the first 10 years after onset of diabetes mellitus had significantly higher plasma level of $\operatorname{sST} 2(\mathrm{n}=21 ; 1012$ [773-1493] pg/ml) at time of onset of diabetes mellitus when compared to patients that did not develop DN ( $\mathrm{n}=199 ; 723$ [4491084] $\mathrm{pg} / \mathrm{ml} ; \mathrm{p}=0.006$ ). This increase in sST2 levels was even more prominent when taking the severity of DN into account since patients that developed macroalbuminuria had the highest plasma levels $(\mathrm{n}=5 ; 1629$ [1416-1849] pg/ml) compared to patients that developed

Table 1 Biochemical and clinical data for study participants, aged 15-34 at onset of diabetes, with or without development of diabetic nephropathy (DN) within 10 years after onset of diabetes

\begin{tabular}{llll}
\hline & No nephropathy $(\mathbf{n}=\mathbf{1 9 9})$ & Nephropathy $(\mathbf{n}=\mathbf{2 1})$ & $\mathbf{p}$ value \\
\hline SST2 $(\mathrm{pg} / \mathrm{ml})$ & $723[449-1084]$ & $1012[777-1493]$ & 0.006 \\
SCD163 $(\mathrm{ng} / \mathrm{ml})$ & $287[221-386]$ & $346[299-450]$ & 0.058 \\
BMl $\left(\mathrm{kg} / \mathrm{m}^{2}\right)$ & $25.1 \pm 3.5$ & $25.1 \pm 4.1$ & 0.911 \\
Age $($ years & $25.0 \pm 5.5$ & $26.7 \pm 6.1$ & 0.181 \\
HbA1c $(\mathrm{mmol} / \mathrm{mol}(\%))$ & $66.1 \pm 15.6(8.2 \pm 1.4)$ & $74.0 \pm 17.2(8.9 \pm 1.6)$ & 0.031 \\
Sex (men/women) & $118 / 81$ & $15 / 6$ & 0.397 \\
Type 1/type 2 diabetes & $122 / 77$ & $9 / 12$ & 0.160 \\
\hline
\end{tabular}


microalbuminuria $(\mathrm{n}=16 ; 917$ [704-1108] pg/ml; $\mathrm{p}=0.002$ ). No differences in sST2 levels at onset of diabetes were found between participants who later developed DR and those who did not $(\mathrm{p}=0.54)$. Plasma levels of sST2 were significantly higher in men (900 [597-1216] $\mathrm{pg} / \mathrm{ml}$ ) than in women (596 [342-777] pg/ml; $\mathrm{p}<0.0001)$. No differences in sST2 levels were found between smokers and non-smokers $(\mathrm{p}=0.65)$ or between patients with type 1 diabetes and type 2 diabetes $(\mathrm{p}=0.98)$. No statistically significant correlation was found between sST2 and BMI although a tendency was observed ( $\mathrm{rs}=0.13$; $\mathrm{p}=0.06$ ). No correlation between plasma levels of sST2 and age ( $\mathrm{rs}=-0.08 ; \mathrm{p}=0.22$ ) or HbA1c ( $\mathrm{rs}=-0.0006$; $\mathrm{p}=0.99)$ was found.

Plasma levels of sST2 and sCD163 levels were strongly correlated ( $r s=0.23 ; \mathrm{p}=0.002$ ). There was also a tendency of higher sCD163 levels in patients that later developed $\mathrm{DN}$ when compared to those who did not $(p=0.058)$. Levels of sCD163 did not correlate significantly with age ( $\mathrm{rs}=-0.05 ; \mathrm{p}=0.51)$, $\mathrm{BMI}(\mathrm{rs}=0.08$; $\mathrm{p}=0.23$ ) or HbA1c levels ( $\mathrm{rs}=-0.05 ; \mathrm{p}=0.51)$, nor did it differ between smokers and non-smokers $(\mathrm{p}=0.74)$, men vs. women $(\mathrm{p}=0.45)$ or between patients with type 1 and type diabetes $(\mathrm{p}=0.27)$.

Levels of HbA1c differed significantly $(\mathrm{p}<0.0001)$ between controls $[60 \mathrm{mmol} / \mathrm{mol}(7.6 \%)]$, and patients with DR only $[72.5 \mathrm{mmol} / \mathrm{mol}(8.8 \%)]$, and concomitant DR and DN [81 mmol/mol (9.6\%)], but not DN only $[61.5 \mathrm{mmol} / \mathrm{mol}(7.8 \%)](\mathrm{p}=0.49)$. No statistically significant differences between the four complication groups regarding BMI $(\mathrm{p}=0.84)$, sex $(\mathrm{p}=0.44)$, age $(\mathrm{p}=0.11)$, smoking $(\mathrm{p}=0.95)$ or diabetes classification $(\mathrm{p}=0.28)$ were observed (Table 1), thereby reducing their possible confounding effects.

Patients diagnosed with type 1 diabetes had lower BMI $(24.5 \pm 3.1)$ compared to patients diagnosed with type 2 diabetes $(25.9 \pm 3.9)(\mathrm{p}=0.005)$ as expected. Patients diagnosed with type 1 diabetes were also slightly younger $(24.3 \pm 5.4$ years $)$ compared to type 2 diabetes $(26.4 \pm 5.8$ years; $\mathrm{p}=0.008)$. Plasma levels of $\mathrm{sST} 2$ and sCD163 did not differ between patients diagnosed with type 1 and type 2 diabetes. No difference in complication development was found between patients with type 1 and type 2 diabetes $(p=0.86)$. A frequency of $6.9 \%$ of patients with type diabetes and $13.5 \%$ of patients with type 2 diabetes developed $\mathrm{DN}(\mathrm{p}=0.11)$.

\section{Discussion}

The main and novel finding in this study is that plasma sST2 levels were significantly higher and at the time of onset of diabetes in subjects who early on, within 10 years, developed nephropathy. Levels of sCD163 were also higher in the same group although did not reach statistical significance. Furthermore, levels of sST2 increased significantly with degree of albuminuria and thus the severity of nephropathy. This significant difference in plasma sST2 levels remained after adjusting for sex, age and BMI. However, levels of sST2 did not differ between patients developing DR or complications in general when compared to controls. Thus, sST2 could be a promising candidate in a future panel identifying patients at high risk of developing particularly renal involvement and diabetes nephropathy.

A strength with the study is that we have a well-defined cohort with young adult subjects and a long follow up time of 10 years making it possible to longitudinally identify complication development. Another strength is that we used commercially available assays with high sensitivity and low variation. A limitation of the study is that relatively few patients developed nephropathy within the follow up period which impacts the statistical power. Most diabetes related complications, DN in particular, occur 15-25 years after onset of diabetes [34]. It is thus possible that the patients in this study may be a subgroup of patients with early onset of nephropathy.

Plasma sST2 levels were not significantly different between men and women, type 1 and type 2 diabetes or smokers and non-smokers. The fact that SST2 levels were not associated with $\mathrm{HbA1c}$ levels, in combination with the high stability of sST2 in plasma and its general robustness makes SST2 a promising predictive biomarker for the development of DN. This is highly interesting since it raises the question of how the pathogenesis of DN and DR may differ from each other, as well as the potential role of SST2 in DN pathogenesis. This is also supported by the fact that not all patients with DN have concomitant retinopathy. This might seem like an unexpected finding, but it is well in line with previous studies $[35,36]$.

Levels of HbA1c were found to be significantly higher in patients who within 10 years developed diabetes related complications, DN and DR taken together. This is expected since HbA1c is a well-known predictive marker for development diabetes-related complication [34, 37]. Interestingly, subjects developing $\mathrm{DN}$ without concomitant DR did not have higher HbA1c levels compared to the control group. Patients with DR had significantly higher HbA1c levels when compared to the control group and DN only. Previous studies have found significantly higher HbA1c levels in patients developing long-term complications such as DN when measuring long term mean HbA1c from onset of diabetes, or at the time of onset of DN $[34,37,38]$. This suggests that sST2 and $\mathrm{HbA} 1 \mathrm{c}$ might complement each other as predictive markers. Analyzing both markers simultaneously may enable a higher precision [39]. 
This study found a tendency of difference in sCD163 plasma levels between patients that did and did not develop nephropathy during the follow up period. Determining the relevance of this tendency would require further studies in larger populations. Previous studies have found associations between sCD163 levels and onset of type 2 diabetes [29] as well as its ligand sTWEAK in type 1 diabetes and complications [40]. To our knowledge associations between sCD163 and DN/DR have not previously been investigated or described.

We found no difference in plasma levels of sCD163 between men and women, type 1 and type 2 diabetes or smokers and non-smokers. Neither was any correlation between sCD163 levels and age or HbA1c found. There was no statistically significant correlation between sCD163 and BMI. This may at first glance seem surprising since high BMI often correlate with higher percentage of body fat, which in turn is associated with higher macrophage infiltration to fatty tissues as well as inflammation and macrophage activation [25, 41]. However, since our study population constitutes of younger individuals with type 1 diabetes being more common than type 2 , the inflammatory mechanism behind the release of sCD163 might be different and the association with BMI of less significance.

Additional larger and prospective longitudinal studies on sST2 and sCD163 levels and development of nephropathy are needed, in both blood and urine. Preferably over a longer follow up period are needed to prove the correlation. We observed that $6.9 \%$ of the patients with type 1 diabetes and $13.5 \%$ of patients with type 2 diabetes developed DN. Although not reaching statistical significance this difference should be investigated in a larger cohort of patients at different ages. Additionally, there is room for further explorations regarding sST2's possible role in the pathogenesis of $\mathrm{DN}$ as well as a possible target for potential therapies.

\section{Conclusions}

In conclusion, there was a strong association between higher plasma levels of sST2 at clinical onset and development of DN within 10 years. With sST2 being highly stable and easily quantified it holds promise to be included in a biomarker panel for later DN development but have far too limited sensitivity and specificity to be used by its own. It is probable that more subjects would develop DN within a longer follow up period which might increase the sensitivity and specificity of the marker. It would therefore be of interest to study the predictive properties of SST2 on a later onset of complication development. To our knowledge, this is the first study in which any association between levels of sST2 or sCD163 with later development of DN has been assessed.

\section{Authors' contributions}

MS analysed the data, interpretation of results, writing parts of the manuscript and critically reviewing the manuscript. JD optimization of the methods, interpretation of results and critically reviewing the manuscript. MKS patient recruitment, parts of the study design and critically reviewing the manuscript. MLO patient recruitment, parts of the study design, responsible for attaining ethical approval and critically reviewing the manuscript. MH parts of the study design, interpretation of results, writing parts of the manuscript and critically reviewing the manuscript. All authors read and approved the final manuscript.

\section{Author details \\ ${ }^{1}$ Department of Clinical Sciences, Diabetes Research Laboratory, Lund University, BMC, B11, 22184 Lund, Sweden. ${ }^{2}$ Department of Medical Sciences, Uppsala University, Uppsala, Sweden. ${ }^{3}$ Department of Endocrinology, Skåne University Hospital Lund, Lund, Sweden.}

\section{Acknowledgements}

The authors would like to thank Mrs. Birgitte Ekholm, Mr. Carl Olsson and Mr. Erik Olsson for excellent technical assistance. The member of DISS study group during collection of samples were Dr. Hans Arnqvist, Linköping; Dr. Jan Bolinder, Stockholm; Dr. Jan W Eriksson, Dr. Lennarth Nyström, Umeå and Dr. Jan Östman, Stockholm.

\section{Competing interests}

The authors declare that they have no competing interests.

\section{Ethics, consent and permissions}

The study was reviewed and approved by the Regional Ethical Review Board in Lund (LU-176-03 and 2009-423), the collection of samples was in accordance with the requirements at the time for sampling and conducted according to the Declaration of Helsinki. Informed consent was obtained from the participants and guardian in case patients are minors before entering the DISS study.

The data analysed in this study is available upon reasonable request from the corresponding author.

\section{Funding}

The study was funded by the Swedish Medical Research Council.

\section{Publisher's Note}

Springer Nature remains neutral with regard to jurisdictional claims in published maps and institutional affiliations.

Received: 8 February 2017 Accepted: 17 May 2017

Published online: 25 May 2017

References

1. Dcct Edic research group. Effect of intensive diabetes treatment on albuminuria in type 1 diabetes: long-term follow-up of the Diabetes control and complications trial and epidemiology of diabetes interventions and complications study. Lancet Diabetes Endocrinol. 2014;2(10):793-800.

2. Hata J, Arima H, Rothwell PM, Woodward M, Zoungas S, Anderson C, Patel A, Neal B, Glasziou P, Hamet P, et al. Effects of visit-to-visit variability in systolic blood pressure on macrovascular and microvascular complications in patients with type 2 diabetes mellitus: the ADVANCE trial. Circulation. 2013;128(12):1325-34.

3. Selvarajah S, Uiterwaal CS, Haniff J, van der Graaf Y, Visseren FL, Bots ML, Group Ss. Renal impairment and all-cause mortality in cardiovascular disease: effect modification by type 2 diabetes mellitus. Eur J Clin Investig. 2013;43(2):198-207

4. Ismail-Beigi F, Craven TE, O'Connor PJ, Karl D, Calles-Escandon J, Hramiak I, Genuth S, Cushman WC, Gerstein HC, Probstfield JL, et al. Combined intensive blood pressure and glycemic control does not produce an additive benefit on microvascular outcomes in type 2 diabetic patients. Kidney Int. 2012;81(6):586-94.

5. Beulens JW, Patel A, Vingerling JR, Cruickshank JK, Hughes AD, Stanton A, Lu J, Thom SM, Grobbee DE, Stolk RP, et al. Effects of blood pressure 
lowering and intensive glucose control on the incidence and progression of retinopathy in patients with type 2 diabetes mellitus: a randomised controlled trial. Diabetologia. 2009;52(10):2027-36.

6. Krolewski AS. Progressive renal decline: the new paradigm of diabetic nephropathy in type 1 diabetes. Diabetes Care. 2015;38(6):954-62.

7. Gansevoort RT, Matsushita K, van der Velde M, Astor BC, Woodward M, Levey AS, de Jong PE, Coresh J, Chronic Kidney Disease Prognosis C. Lower estimated GFR and higher albuminuria are associated with adverse kidney outcomes. A collaborative meta-analysis of general and high-risk population cohorts. Kidney Int. 2011;80(1):93-104.

8. Svensson MK, Cederholm J, Eliasson B, Zethelius B, Gudbjornsdottir S, Swedish National Diabetes R. Albuminuria and renal function as predictors of cardiovascular events and mortality in a general population of patients with type 2 diabetes: a nationwide observational study from the Swedish National Diabetes Register. Diabetes Vasc Dis Res. 2013;10(6):520-9.

9. Bjornstad P, Cherney DZ, Snell-Bergeon JK, Pyle L, Rewers M, Johnson RJ, Maahs DM. Rapid GFR decline is associated with renal hyperfiltration and impaired GFR in adults with type 1 diabetes. Nephrol Dial Transplant. 2015;30(10):1706-11.

10. Caramori ML, Parks A, Mauer M. Renal lesions predict progression of diabetic nephropathy in type 1 diabetes. J Am Soc Nephrol. 2013;24(7):1175-81

11. Hojs R, Ekart R, Bevc S, Hojs N. Biomarkers of renal disease and progression in patients with diabetes. J Clin Med. 2015;4(5):1010-24.

12. Kern EF, Erhard P, Sun W, Genuth S, Weiss MF. Early urinary markers of diabetic kidney disease: a nested case-control study from the diabetes control and complications trial (DCCT). Am J Kidney Dis. 2010;55(5):824-34.

13. Nielsen SE, Sugaya T, Hovind P, Baba T, Parving HH, Rossing P. Urinary liver-type fatty acid-binding protein predicts progression to nephropathy in type 1 diabetic patients. Diabetes Care. 2010;33(6):1320-4.

14. Ahsan H. Diabetic retinopathy-biomolecules and multiple pathophysiology. Diabetes Metab Syndr. 2015;9(1):51-4.

15. Lee R, Wong TY, Sabanayagam C. Epidemiology of diabetic retinopathy, diabetic macular edema and related vision loss. Eye Vis (Lond). 2015;2:17.

16. Milovanovic M, Volarevic V, Radosavljevic G, Jovanovic I, Pejnovic N, Arsenijevic N, Lukic ML. IL-33/ST2 axis in inflammation and immunopathology. Immunol Res. 2012;52(1-2):89-99.

17. Bandara G, Beaven MA, Olivera A, Gilfillan AM, Metcalfe DD. Activated mast cells synthesize and release soluble ST2-a decoy receptor for IL-33. Eur J Immunol. 2015;45(11):3034-44.

18. Miller AM, Purves D, McConnachie A, Asquith DL, Batty GD, Burns H, Cavanagh J, Ford I, McLean JS, Packard CJ, et al. Soluble ST2 associates with diabetes but not established cardiovascular risk factors: a new inflammatory pathway of relevance to diabetes? PLOS ONE. 2012;7(10):e47830.

19. Fousteris E, Melidonis A, Panoutsopoulos G, Tzirogiannis K, Foussas S, Theodosis-Georgilas A, Tzerefos S, Matsagos S, Boutati E, Economopoulos T, et al. Toll/interleukin-1 receptor member ST2 exhibits higher soluble levels in type 2 diabetes, especially when accompanied with left ventricular diastolic dysfunction. Cardiovasc Diabetol. 2011;10:101.

20. Dieplinger B, Egger M, Haltmayer M, Kleber ME, Scharnagl H, Silbernagel G, de Boer RA, Maerz W, Mueller T. Increased soluble ST2 predicts longterm mortality in patients with stable coronary artery disease: results from the Ludwigshafen risk and cardiovascular health study. Clin Chem. 2014;60(3):530-40.

21. Vilchez JA, Perez-Cuellar M, Marin F, Gallego P, Manzano-Fernandez S, Valdes M, Vicente V, Noguera-Velasco JA, Lip GY, Ordonez-Llanos J, et al. sST2 levels are associated with all-cause mortality in anticoagulated patients with atrial fibrillation. Eur J Clin Investig. 2015;45(9):899-905.

22. Miller WL, Saenger AK, Grill DE, Slusser JP, Bayes-Genis A, Jaffe AS. Prognostic value of serial measurements of soluble suppression of tumorigenicity 2 and galectin-3 in ambulatory patients with chronic heart failure. J Cardiac Fail. 2016;22(4):249-55.

23. Moller HJ. Soluble CD163. Scand J Clin Lab Investig. 2012;72(1):1-13.
24. Parkner T, Sørensen LP, Nielsen AR, Fischer CP, Bibby BM, Nielsen $S$, Pedersen BK, Moller HJ. Soluble CD163: a biomarker linking macrophages and insulin resistance. Diabetologia. 2012;55(6):1856-62.

25. Fjeldborg K, Christiansen T, Bennetzen M, Moller HJ, Pedersen SB, Richelsen B. The macrophage-specific serum marker, soluble CD163, is increased in obesity and reduced after dietary-induced weight loss. Obesity (Silver Spring, Md). 2013;21(12):2437-43.

26. Vendrell J, Chacon MR. TWEAK: a new player in obesity and diabetes. Front Immunol. 2013:4:488.

27. Akahori H, Karmali V, Polavarapu R, Lyle AN, Weiss D, Shin E, Husain A, Naqvi N, Van Dam R, Habib A, et al. CD163 interacts with TWEAK to regulate tissue regeneration after ischaemic injury. Nat Commun. 2015;6:7792.

28. Abu El-Asrar AM, De Hertogh G, Nawaz Ml, Siddiquei MM, Van den Eynde K, Mohammad G, Opdenakker G, Geboes K. The tumor necrosis factor superfamily members TWEAK, TNFSF15 and fibroblast growth factorinducible protein 14 are upregulated in proliferative diabetic retinopathy. Ophthalmic Res. 2015;53(3):122-30.

29. Moller HJ, Frikke-Schmidt R, Moestrup SK, Nordestgaard BG, TybjaergHansen A. Serum soluble CD163 predicts risk of type 2 diabetes in the general population. Clin Chem. 2011;57(2):291-7.

30. Diabetes mellitus. Report of a WHO Study Group. World Health Organ Tech Rep Ser. 1985; 727:1-113.

31. Svensson M, Sundkvist G, Arnqvist HJ, Bjork E, Blohme G, Bolinder J, Henricsson M, Nystrom L, Torffvit O, Waernbaum I, et al. Signs of nephropathy may occur early in young adults with diabetes despite modern diabetes management: results from the nationwide population-based Diabetes Incidence Study in Sweden (DISS). Diabetes Care. 2003;26(10):2903-9.

32. Mogensen $C E$, Christensen $C K$, Vittinghus $E$. The stages in diabetic renal disease. With emphasis on the stage of incipient diabetic nephropathy. Diabetes. 1983;32(Suppl 2):64-78.

33. Weir RA, Miller AM, Murphy GE, Clements S, Steedman T, Connell JM, McInnes IB, Dargie HJ, McMurray J. Serum soluble ST2: a potential novel mediator in left ventricular and infarct remodeling after acute myocardial infarction. J Am Coll Cardiol. 2010;55(3):243-50.

34. Nordwall M, Abrahamsson M, Dhir M, Fredrikson M, Ludvigsson J, Arnqvist HJ. Impact of HbA1c, followed from onset of type 1 diabetes, on the development of severe retinopathy and nephropathy: the VISS Study (Vascular Diabetic Complications in Southeast Sweden). Diabetes Care. 2015;38(2):308-15.

35. Chavers BM, Mauer SM, Ramsay RC, Steffes MW. Relationship between retinal and glomerular lesions in IDDM patients. Diabetes. 1994:43(3):441-6.

36. Lovestam-Adrian M, Agardh E, Agardh CD. The incidence of nephropathy in type 1 diabetic patients with proliferative retinopathy: a 10-year followup study. Diabetes Res Clin Pract. 1998;39(1):11-7.

37. Tannus LR, Drummond KR, Clemente EL, da Matta Mde F, Gomes MB, Brazilian Type 1 Diabetes Study G. Predictors of cardiovascular autonomic neuropathy in patients with type 1 diabetes. Front Endocrinol. 2014;5:191.

38. Nathan DM, McGee P, Steffes MW, Lachin JM, Group DER. Relationship of glycated albumin to blood glucose and $\mathrm{HbA} 1 \mathrm{c}$ values and to retinopathy, nephropathy, and cardiovascular outcomes in the DCCT/EDIC study. Diabetes. 2014;63(1):282-90.

39. Parikh R, Mathai A, Parikh S, Chandra Sekhar G, Thomas R. Understanding and using sensitivity, specificity and predictive values. Indian J Ophthalmol. 2008;56(1):45-50.

40. Llauradó G, González-Clemente J-M, Maymó-Masip E, Subías D, Vendrell J, Chacón MR. Serum levels of TWEAK and scavenger receptor CD163 in type 1 diabetes mellitus: relationship with cardiovascular risk factors. A case-control study. PLoS ONE. 2012;7(8):e43919.

41. Al-Daghri NM, Al-Attas OS, Bindahman LS, Alokail MS, Alkharfy KM, Draz HM, Yakout S, McTernan PG, Sabico S, Chrousos GP. Soluble CD163 is associated with body mass index and blood pressure in hypertensive obese Saudi patients. Eur J Clin Investig. 2012;42(11):1221-6. 